ENSAYOS

\title{
EL COMPONENTE INVESTIGATIVO Y LA FORMACION DOCENTE EN VENEZUELA
}

\author{
Research and teaching practice in education of teachers
}

\section{Natalia Bondarenko Pisemskaya}

Universidad de Oriente, Núcleo Nueva Esparta, Isla de Margarita, Venezuela. npisemskaya@gmail.com

\section{Resumen}

El propósito del ensayo es presentar el elemento investigativo desde el punto de vista de la transformación educativa, esto es, el tránsito desde un docente como transmisor de datos hacia un docente creativo que busca soluciones a los problemas. Los temas principales que se abordan en el ensayo son: cambio paradigmático en el campo educativo, investigación como proceso integral, práctica docente como base para la formación de docentes-investigadores, entre otros. El ensayo se refiere a los campos teóricos como orientación postpositivista, racionalidad crítica, interacción humana y autoeducación permanente. Se concluye que actualmente se debe formar en y para la investigación, para lo cual, antes que nada, hay que transformar la cultura de las instituciones formadoras de docentes.

Palabras clave: formación docente, enfoque investigativo, prácticas profesionales, formación transdisciplinaria.

\begin{abstract}
The purpose of the essay is to introduce the research element from the point of view of educational transformation, that is, the transition from the conception of a teacher as a transmitter of data, towards a creative teacher who is looking for solutions to the problems. The principal topics covered in the essay are: paradigmatic change in the field of education, research as an integral process, teaching practice as the basis for the education of teachers - investigators, among others. The essay makes reference to the theoretic fields such as postpositivist orientation, critical rationality, human interaction and permanent self - education. It is concluded that currently, it is necessary to educate in and for research, and for that matter, in the first place, it is necessary to transform the culture of the institutions that educate teachers.

Key words: teacher education, research perspective, professional practice, transdisciplinary education.
\end{abstract}


El vínculo entre la formación docente y la investigación es un tema que aún se encuentra poco definido, poco estudiado y, por consiguiente, escasamente instrumentado en los espacios académicos. Esta situación se debe, en parte, al hecho de existir una falta de claridad pedagógica en las respuestas a las dos preguntas fundamentales: a) ¿qué significa ser docente? y b) ¿qué significa investigar? La ausencia de respuestas contundentes a estas preguntas hace aún más difícil entender la relación que debe existir entre ambas. Este vínculo se presenta, en la mayoría de los casos, de manera abstracta, es decir, sin que ello implique modificación de estructuras académicas o prácticas pedagógicas. A pesar de esta situación, actualmente se está notando un creciente interés hacia este tema por parte de investigadores, profesores formadores y hasta los mismos estudiantes (futuros docentes), debido, principalmente, al cambio paradigmático que se está presentando en todos los ámbitos de la sociedad, cambio que se manifiesta a través de la ruptura de las relaciones tradicionales de poder, desvanecimiento del concepto de la verdad, cuestionamiento del método científico, caos conceptual y otros fenómenos afines.

Tradicionalmente, la formación docente ha estado basada en el supuesto de la homogeneidad y en un único modelo de enseñanza, el modelo de transmisión (Morán 2003). Esta concepción estaba orientada por el paradigma positivista y la racionalidad tecnocrática, características de la época Moderna. Desde esta perspectiva, el Universo funciona mecánicamente y las leyes de la naturaleza se presentan como eternas e inmutables. La ontología positivista postula que existe un mundo real, independiente del observador, que se puede aprehender; según la epistemología positivista, el conocimiento es totalmente objetivo y la finalidad de la ciencia es alcanzar la verdad. De acuerdo con esto, el modelo tradicional de formación docente, el cual se enmarcaba dentro de la lógica del paradigma educativo de la Modernidad, se caracterizaba por tener su énfasis en la adquisición y dominio de conocimientos, mientras que el currículum de formación se imponía como fragmentado y regido por la lógica disciplinaria.

La crisis que se hizo parte de todas las esferas de la civilización Moderna, también se ha hecho sentir en la Educación, ya que, naturalmente, factores políticos, económicos, culturales e ideológicos son parte del contexto donde se desarrolla la realidad educativa. Como consecuencia, los viejos modelos de formación docente, "atrapados en las lógicas del pensamiento moderno o pensamiento clásico" y anclados “... en unos referentes externos "universales" (Peñalver 2000), ya no podían satisfacer las nuevas realidades y los cambios que hoy están sucediendo en el mundo en general y en nuestro país en particular. Los fuertes desajustes entre la formación teórica y la realidad educativa, una “...asincronía entre prácticas, discursos y... sentidos portados en un espacio institucional" (Lanz y Fergusson 2005: 16), entre otros factores, llevaron al agotamiento del modelo cientificista, el cual se saturó y terminó siendo una caricatura del reduccionismo y la simplicidad.

Aunque en el día de hoy los modelos de formación docente todavía exhiben una fuerte dependencia de los modelos tecnoeconómicos característicos de la Modernidad, se está haciendo más y más evidente que esta situación los está llevando a una severa crisis. Como señala Morán, "pocos campos como el de la formación docente están tan urgidos de innovación y experimentación" (2003: 134). Por consiguiente, dada esta crisis del discurso educativo de la Modernidad en general y la inoperancia del modelo tradicional de formación docente en particular, se presenta como desafío la necesidad de transitar hacia un nuevo enfoque del proceso de formación docente, cuadro que puede caracteri- 
zarse como tránsito desde la tendencia profesionalizante hacia el enfoque investigativo en la formación docente.

Desde el punto de vista de este nuevo enfoque, la sociedad del conocimiento plantea la formación de los docentes no para acumular conocimientos sino para investigar, pensar, comunicarse, tener iniciativa, aprender a trabajar por su propia cuenta y de esta manera contribuir al desarrollo comunitario. De acuerdo con este planteamiento, el docente debe asumir su papel protagónico en la transformación del sistema educativo; para eso, se requiere de un futuro docente que rompa con la concepción de una simple transmisión del saber y que participe activamente en la investigación de su propia práctica y en la resolución de los problemas que le plantea su entorno social. Como también lo señala Barrios (2000), uno de los elementos principales de la transformación cualitativa del currículum de la formación docente debe ser la incorporación de la investigación como elemento clave para el desarrollo de competencias para el cambio educativo. En este sentido, la investigación educativa ya no puede ser, exclusivamente, cuestión de laboratorio para controlar variables, como lo era antes, sino un proceso creativo, autónomo y autorreflexivo, durante el cual el futuro docente establezca críticas a sus interpretaciones sobre los valores, creencias y costumbres de la comunidad que él investiga.

En el marco de este nuevo enfoque, se ha difundido en América Latina la idea de formación docente asociada con la pedagogía crítica y con la investigación desde la práctica, que valora la capacidad de los futuros docentes de construir sus propios conocimientos y que postule esa "enseñanza reflexiva" como una propuesta múltiple que integre la enseñanza, el aprendizaje y la investigación.

Indudablemente, la enseñanza por investigación posee varias ventajas: contribuye al desarrollo profesional del formador y promueve el aprendizaje funcional de los futuros docentes, potencia el trabajo en equipo y permite conformar un currículum integrado, basado en el estudio de los problemas que son vitales para la comunidad educativa.

Sin embargo, la enseñanza por investigación presenta serios desafíos para todos los involucrados, porque implica el abandono del papel tradicional que desempeña el docente en una aula de clase, se opone al currículum organizado por asignaturas y es bastante difícil de planificar. Como obstáculo más importante para el desarrollo de la enseñanza por investigación, se presenta el desconocimiento práctico de la dinámica de este tipo de enseñanza, es decir, la condición de los formadores que no poseen experiencia y preparación en esta materia. En resumidas cuentas, por los momentos, la enseñanza por investigación se visualiza como una alternativa poco conocida y desarrollada en la práctica.

Como punto de partida para promover la enseñanza por investigación, los docentes deberían preguntarse a sí mismos si quieren formar a sus estudiantes para ser usuarios del conocimiento de otros o para construir su propio conocimiento, a partir de sus experiencias. Una vez respondida esta pregunta, la cuestión de fondo consiste en qué tipo de relación se busca establecer entre la investigación y los procesos de construcción del conocimiento en cada campo incluido en el currículum. En otras palabras, la tarea de los formadores es cómo ubicar y tratar curricularmente a la investigación. Esta tarea se resuelve, en la gran mayoría de los casos, en la incorporación de una serie de asignaturas sobre los métodos y técnicas de investigación en el currículum de formación, sin ir más allá. En otros casos, se llega al otro extremo: la manera de concebir la investigación crea cierta ambigüedad respecto de si se está formando docentes o investigadores. Todo esto demuestra una vez más que las fronteras entre formar, enseñar e investigar se están 
borrando rápidamente, haciéndose más y más difusas. Tanto así que Müller (1995) afirma que la investigación en sí es un proceso pedagógico.

En Venezuela, el proceso de cambio hacia el nuevo enfoque en la formación docente apenas está comenzando, y al respecto existen muchas dificultades e incertidumbres. Anteriormente, ya se mencionó que no existe una respuesta unívoca a la pregunta ¿qué significa investigar? En este sentido, en algunos docentes venezolanos todavía se tiene una idea bastante vaga al respecto, y tampoco se ha concientizado bien la importancia de la investigación en el desarrollo de Venezuela como nación. Así, Níaz afirma que en nuestro país "la participación en investigación arbitrada por pares y publicación en revistas indizadas se encuentra a un nivel preocupante" (2000: 38). Lo cierto es que la riqueza de una nación se determina, entre otras cosas, por su riqueza científica, o investigativa. Al respecto, el mismo autor señala que “... a nivel internacional cada día se reconoce más que hay una relación estrecha... entre el desarrollo económico de una nación y la productividad de sus investigadores..." (ibid). Por eso, es lamentable que en las instituciones de educación superior venezolanas todavía no existe una verdadera cultura de investigación, aunque no se puede negar que se están haciendo buenos avances en esta dirección y, como sociedad, finalmente hemos comenzado a valorar las actividades de investigación científica.

Por esta razón, no es de extrañar que exista todavía una cierta desorientación respecto a la formación investigativa de los docentes. A pesar del consenso de que no puede haber separación entre lo que se enseña y aquello que se conoce sobre un fenómeno, son todavía muy pocos los países en América Latina que hayan logrado que los centros de formación docente realicen o reciban investigación docente. En este sentido, Morín de Valero señala sin titubear que “... no hay ningún país latinoamericano que ponga a la investigación como componente de la formación docente inicial -sólo Argentina que lo está logrando con desigual éxito" (2006: 3). En cuanto a nuestro país, se puede afirmar que en Venezuela, la incorporación del componente investigativo en el proceso de la formación docente apenas se inició recientemente. Al respecto, cabe mencionar, por ejemplo, la propuesta de Díaz (2004), la cual consiste en agregar a los cuatro componentes necesarios para la formación docente (el científico, el psicopedagógico, el cultural y el de la práctica docente) dos componentes más: la investigación y la formación de un pensamiento curricular, o también el hecho de que en las páginas web de la Universidad Pedagógica Experimental Libertador y de la Universidad de Oriente, aparece el componente investigativo como uno de los ejes transversales de la formación docente inicial, lo cual significa que se está empezando a prestar debida atención a este componente y se está intentando realizar la preparación correspondiente de los estudiantes, futuros docentes.

A pesar de estos avances, el desajuste entre la teoría y la práctica investigativa aún es evidente, y es por eso que, cuando Morín de Valero afirma que en Venezuela “... no existe ninguna institución universitaria que proporcione una completa formación profesional, indispensable para que los docentes universitarios puedan manejar la docencia con la investigación" (2006:4), la autora se está refiriendo, antes que nada, a la incongruencia que existe todavía entre la incorporación teórica del componente investigativo en el currículum de formación y la situación real, carente de actividades investigativas significativas por parte de los estudiantes, actividades que podrían llevarlos a presentar los resultados parciales o totales de sus investigaciones en eventos nacionales e internacionales, o a la publicación de artículos y ensayos en revistas indizadas y arbitradas. (M. Morín de Valero, conversación telefónica, febrero 15, 2008). 
Cabe destacar que, desde el punto de vista legal, la formación investigativa de los docentes en Venezuela se encuentra bien sustentada en los documentos oficiales, entre ellos, la Ley Orgánica de Educación (1980), la cual postula que la Universidad tiene entre sus funciones fomentar la investigación de nuevos conocimientos e impulsar el progreso de la ciencia. Desde esta misma perspectiva, la Conferencia Mundial sobre la Educación Superior (1998) proclama entre las funciones y misiones de la educación superior la función de educar, formar y realizar investigaciones.

Volviendo al tema de la incorporación de facto de la formación investigativa en el currículum de la formación docente, ésta se basa en las siguientes consideraciones, cónsonas con los postulados epistemológicos del paradigma postpositivista:

1. La experiencia se presenta como fuente de conocimiento para el educador;

2. El proceso de conocer implica curiosidad, equivocación, acierto, sufrimiento, satisfacción, alegría, el despliegue de lo lúdico, lo estético, lo sensible, lo imaginativo;

3. Todo conocimiento es, antes que nada, ético, ya que en él están implicados respeto, dignidad humana, autonomía, diversidad, compromiso;

4. Todo conocimiento es inacabado, tentativo y sujeto a revisión.

El componente investigativo, según Sánchez, debe ser "un proceso integral en el que no sólo el adelanto científico tiene lugar o está presente, sino y más importante, la construcción de la subjetividad creativa como entidad que está en capacidad de hacerlo" (2006: 3). Ahora, para la construcción de esta subjetividad creativa es imprescindible desarrollar en los futuros docentes un conjunto de habilidades, en el cual se destacan: pensar, crear, diseñar, resolver, interactuar, manejar, usar, producir y comunicar. Para el desarrollo de estas y otras habilidades, los estudiantes deben recibir una formación investigativa integral, basada en dos vertientes principales, una teórica y la otra práctica: a) dominio de los principios y métodos de la investigación y b) participación en la dirección y ejecución de la propia investigación. En cuanto a los tipos de investigación a desarrollar, los docentes en formación podrían optar bien sea por la investigación -acción, investigación de campo, estudio de casos, proyecto factible, etc., tomando decisiones también respecto a la modalidad de investigación que deseen realizar- individual o grupal, con grupos oficiales o particulares, resultando o no en publicaciones de diversa índole. Pero, por encima de todo, hay que enfatizar la importancia del derecho del estudiante de tener la libertad para escoger el tópico a investigar, siempre y cuando éste esté enmarcado dentro de los campos indagatorios privilegiados, de las líneas de investigación prioritarias, o de los lineamientos de la política nacional de investigación científica. Sobre este particular, Scharifker afirma que "...lo que hay que investigar en las Universidades es todo, prácticamente no hay nada que queda fuera del campo de acción de las Universidades. Hay que investigar lo inútil, lo que uno no pueda ver a primera vista o una utilidad inmediata, eso debe ser investigado" (1995: 56).

Desde el punto de vista más concreto, y cónsono con las exigencias de la sociedad contemporánea, una formación docente investigativa implicaría apartar tiempo necesario para la reflexión, constituir e integrar grupos de investigación, organizar las actividades académicas de manera más flexible. Las mismas actividades de investigación podrían concretarse en proyectos integrados, orientados hacia la comunidad, los cuales trabajarían en dos direcciones principales: a) mejoramiento del proceso enseñanza-aprendizaje 
y b) acciones hacia el entorno, para dar respuestas satisfactorias a los problemas que demandan los sectores sociales.

La forma más común de llevar a cabo la investigación en los institutos de formación es a través de los talleres de la práctica docente y el entrenamiento en las técnicas de investigación-acción, entendida esta última como una investigación sistemática hecha por los futuros docentes sobre sus propias prácticas. En este tipo de investigación, las propias comunidades educativas proponen cambios en el aula y en la escuela mediante la utilización de la investigación; en ella, la teoría y la práctica se unen para generar el cambio y crear una teoría educativa a partir de la comprensión, reflexión y acción. A propósito, Zeichner y Diniz-Pereira (2006) comentan que la participación de los educadores en proyectos de investigación-acción puede transformarlos en "consumidores" más críticos del conocimiento educativo generado en las universidades.

En este sentido, las prácticas profesionales deberían constituir el vehículo articulador para la reflexión en y durante el proceso de la formación, a fin de fortalecer la relación entre la teoría y la práctica educativa, lo cual implica la posibilidad de reconstruir la teoría desde el campo de la acción. Las prácticas posibilitan a los estudiantes a comprender la multidimensionalidad de la enseñanza y a crecer profesionalmente. Bedoya invita a "entender la práctica docente como una propuesta de investigación, como articulación de proyectos de investigación, como procesos de comprensión y transformación de la misma realidad educativa" (2000: 32), procesos que van desde el planteamiento de los problemas hasta la reflexión y búsqueda de posibles soluciones. Las prácticas implican el desarrollo de capacidades de observación, descripción e interpretación en su propio contexto, con el fin de comprender mejor la realidad escolar, revisarla críticamente y transformarla. También Sayago y Chacón señalan que "las Prácticas son realmente prácticas cuando se convierten en oportunidades para investigar y proponer mejoras de la enseñanza, en procesos de observación, planificación, acción y revisión de las acciones" (2006: 60). Aquí se trata de lo que Carr y Kemmis (1988) llaman "una espiral autorreflexiva". El profesor -supervisor externo de las prácticas- se convierte en este proceso en un colaborador, en un coinvestigador activo, quien apoya al estudiante cuando éste lo necesite, rompiendo con la concepción de la simple transmisión de saberes y pasando al diálogo constante profesor-alumno. De allí la necesidad urgente de impulsar en y desde las prácticas procesos de investigación en los espacios escolares, los cuales se convierten en estímulos para la investigación en la enseñanza, investigación-acción, una herramienta valiosa para el desarrollo personal y profesional, para la autoeducación y actualización permanente posterior, o "en servicio", del docente.

De esta manera, la investigación se presenta en las universidades y en los institutos de formación docente como:

1. Eje de formación docente, la cual integraría todos los contenidos de la formación;

2. Modelo de formación docente, la cual desplazaría la rigidez de los textos y el totalitarismo de los programas;

3. Instrumento para la formación docente, generando un cambio desde un informador de clase a un coinvestigador del conocimiento que requieren los estudiantes;

4. Metodología didáctica para la formación de estudiantes-investigadores. 
Resumiendo la importancia de la formación investigativa, se puede afirmar que en este contexto la investigación adquiere un carácter transversal, impregnando todas las actividades académicas de los futuros docentes, atravesándolas como eje articulador del currículum, contribuyendo de esta manera a su formación integral y transdisciplinaria.

Más importante aún, con la incorporación del componente investigativo se invierten las relaciones tradicionales de poder en las universidades e institutos de formación, pasando el poder desde el profesor formador al estudiante que se está formando, el cual toma el control de su propio aprendizaje y empieza a desempeñar el papel protagónico desde su acción investigativa.

Para hacer realidad la formación investigativa de los docentes es necesario, antes que nada, transformar la cultura de las instituciones formadoras, por lo cual se recomienda a las universidades y a los institutos de formación lo siguiente:

1. Destinar tiempo, espacio y recursos para la formación investigativa;

2. Modernizar el currículum y actualizar los programas de formación, incorporando el componente investigativo de manera transversal en los mismos, como parte de la formación académica;

3. Promover las relaciones estrechas con los planteles, al igual que con los sectores empresariales y productivos del país, concretando apoyos a fin de elevar los niveles de investigación.

Por su parte, los formadores de los formadores deben redefinir su rol como entes transformadores del cambio educativo, inspirados en principios democráticos y de justicia social, reconociendo la importancia de la función de investigación y dejando de ser receptores pasivos de los avances científicos, logrados en otros países, para convertirse en agentes activos, críticos y creadores del conocimiento, participando activamente en procesos de transformación social a través de la integración de la docencia y la investigación.

Finalmente, y a manera de conclusión, en la época actual, dada la complejidad de la sociedad y la crisis que se está viviendo en materia educativa, es necesario replantear la formación de los futuros docentes en el área de la investigación, integrando de esta manera las dos funciones fundamentales e interrelacionadas: docencia e investigación.

\section{BIBLIOGRAFIA}

Barrios, O. (2000). Formación docente: teoría y práctica. [Artículo en línea]. Disponible en: http:// www.umce.cl/cip_publica_tesis_pedagogia _ y_didactica.htm [Consulta: 2008, Febrero 28]

Bedoya, J. (2000). Epistemología y Pedagogía. Madrid: Graó.

Carr, W. y S. Kemmis (1988). Teoría crítica de la enseñanza. Barcelona: Martínez Roca.

Díaz, V. (2004). Currículum, investigación y enseñanza en la formación docente. Caracas: Universidad Pedagógica Experimental Libertador.

Ley orgánica de Educación (1980).

Lanz, R. y Fergusson, A. (2005). La reforma universitaria en el contexto de la mundialización del conocimiento. Caracas: OPUS.

Morán, P. (2003). El vínculo de la docencia y la investigación. México: Plaza y Valdés.

Morín de Valero, M. (2006). Investigación y formación docente. [Artículo en linea]. Disponible en: http://www.orestesenlared.com.ve /Colaboradores/lainvestigacionylaformaciondocente.pdf [Consulta: 2008, Febrero 30] 
Müller, I. (1995). Los orígenes de la Universidad Investigativa. Centro de investigaciones de la Universidad Pedagógica Nacional. Santafé de Bogotá: CIUP.

Níaz, M. (2000). Investigación y la riqueza de una nación. Interciencia 25 (1): 37-40.

Peñalver, L. (2000). La Formación docente en Venezuela: compleja y transdisciplinaria. Tesis doctoral no publicada, Maturín: Universidad Pedagógica Experimental Libertador.

Sánchez, J. (2006). Formación docente e investigación en el contexto de la educación venezolana actual. Ponencia presentada en el I Congreso Nacional Bolivariano Universitario, Trujillo.

Sayago, Z. y M. Chacón (2006). Las prácticas profesionales en la formación docente: hacia un nuevo diario de ruta. Educere 10 (32): 55-66.

Scharifker, B. (1995). La investigación en la universidad experimental en el sistema educativo venezolano. Memorias V Seminario sobre Investigación en las Universidades del País (pp. 54-61). Caracas: CDCHT.

UNESCO (1998). Declaración Mundial para la Educación Superior en el Siglo XXI. Conferencia Mundial sobre la Educación Superior.

Zeichner, K. y J. Diniz-Pereira (2006). Investigación de los educadores y formación docente orientada a la transformación social. Rieda [Revista en línea], 28 (2). Disponible: http://tariacuri. crefal.edu.mx/ crefal/ rieda /jul dic2006/contrapunto [Consulta: Abril 15, 2008] 